\title{
Nível de atividade física: questionário internacional de atividades físicas e tempo de prática em mulheres idosas
}

\author{
Level of physical activity: international physical activity questionnaire and time of \\ practice in older women
}

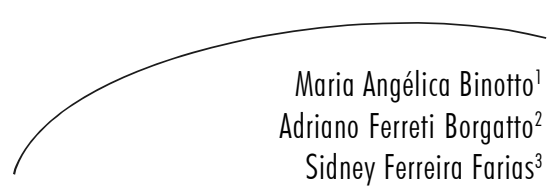

\section{Resumo}

O objetivo do estudo foi verificar o nível de atividade física habitual e o tempo de prática de idosas praticantes de atividades físicas em um grupo de convivência. Este estudo se caracteriza como descritivo de corte transversal. A amostra não probabilística constituiu-se de 234 idosas do sexo feminino com idade igual ou superior a 60 anos $(69,52 \pm 5,51$ anos). Para a coleta de dados, utilizaram-se um questionário com informações referentes à satisfação com a saúde, doenças e uso de medicamentos e o Questionário Internacional de Atividades Físicas (IPAQ) - forma longa, adaptado para idosos. O tempo de prática (anos completos) foi aferido por meio de entrevista individual. Os dados foram analisados por meio do programa estatístico SPSS 12.0, utilizando-se de análises descritivas. No que se refere à prática de atividade física habitual, as idosas, em sua maioria $(82,1 \%)$, foram consideradas mais ativas, com média semanal 322,62 minutos. $\mathrm{Na}$ contribuição dos diferentes domínios da atividade física, quanto ao dispêndio total em minutos por semana de atividades físicas, destacam-se com maior prevalência as atividades de lazer/recreação (42,01\%). Em relação ao tempo de prática das idosas no grupo, observou-se que o tempo médio de participação no grupo foi de $7,35( \pm 5,34)$ anos. Além da implantação de programas de atividades físicas direcionados aos idosos, principalmente para os idosos com idade mais avançada, os programas devem ter como meta a melhora das capacidades físicas do indivíduo e a conscientização da importância da prática das atividades físicas como fator de melhoria da qualidade de vida.

\section{Abstract}

This study aimed to determine the level of habitual physical activity and time of practice of older practitioners of physical activities in a acquaintance group. It is a cross-sectional descriptive and the non-probability sample consisted of 234 elderly females aged more than 60 years (69.52 \pm 5.51 years). For data collection, it was used

\footnotetext{
Universidade Estadual do Centro-Oeste, Departamento de Educação Física. Irati, Brasil.

2 Universidade Federal de Santa Catarina, Centro Tecnológico, Departamento de Informática e Estatística. Florianópolis, SC, Brasil.

3 Universidade Federal de Santa Catarina, Centro de Desportos, Departamento de Educação Física. Florianópolis, SC, Brasil.
}

Correspondência / Correspondence

Maria Angélica Binotto

Rua André Filipak, 160/05

84800-000 - Irati, PR, Brasil

E-mail: manbinotto@yahoo.com.br
Palavras-chave:

Envelhecimento. Atividade

Física. Grupo de

Convivência. Estudo

Transversal. 
a questionnaire with information related to satisfaction with health, disease and medication use and the International Physical Activity Questionnaire (IPAQ) - long form, adapted for the elderly. The time of practice (full years) was informed through individual interviews. Data were analyzed using the statistical program SPSS 12.0, with descriptive analysis. Regarding the practice of physical activity, the elderly, most $(82.1 \%)$, were more active with weekly average of 322.62 minutes. In the contribution of different domains of physical activity in the total expenditure in minutes per week of physical activity, the most prevalent domain were the leisure activities and recreation (42.01\%). Regarding the time of practice in the elderly group, the average time of participation in the group was $7.35( \pm 5.34)$ years. Besides the deployment of physical activity programs targeted to seniors, especially for the elderly with advanced age, the programs must have a goal to improve the physical capabilities of the individual and awareness of the importance of the practice of physical activities as a factor to improve the quality of life.
Key words: Aging. Physical Activity. Group Interaction. Crosssectional Study.

\section{INTRODUÇÃOO}

O envelhecimento tornou-se um dos fenômenos que mais se evidenciam nas sociedades atuais. Está bem documentado na literatura científica ${ }^{1-5}$ que o processo de envelhecimento em idades avançadas está associado a alterações físicas, fisiológicas, psicológicas e sociais, bem como ao surgimento de doenças crônico-degenerativas advindas de hábitos de vida inadequados (tabagismo, ingestão alimentar incorreta, tipo de atividade laboral, ausência de atividade física regular), que se refletem na redução da capacidade para realização das atividades da vida diária.

A importância e os benefícios dos exercícios físicos nestapopulação sãocadavez mais evidentes eas pesquisas têm demonstrado o quanto eles podem minimizar a degeneração provocada pelo envelhecimento e contribuir para a qualidade de vida do idoso, sobretudo em relação às atividades da vida diária influenciando na dependência e na autonomia do idoso. ${ }^{25,6}$. No entanto, no Brasil o sedentarismo apresenta alta prevalência, causando custos elevados, tanto diretos quanto indiretos, para o sistema de saúde ${ }^{7,8}$.

Estudos de caráter epidemiológico de coorte envolvendo populações idosas demonstram que a manutenção de um hábito de vida ativo está associada à diminuição do risco de quedas e fraturas na região do quadril, ${ }^{9-12}$ redução no declínio da massa muscular e óssea ${ }^{1,13}$ e menor incidência de doenças cardiovasculares, respiratórias, neoplasias e obesidade ${ }^{11,14}$. Sendo assim, os programas de atividades físicas emergem como uma perspectiva de promoção da saúde no processo de envelhecimento.

Nos anos 80 e 90 , houve uma expansão da oferta de programas de atividades físicas e formação de grupos de convivência para idosos. ${ }^{15}$ Atualmente, observa-se que há uma disseminação destes programas direcionados aos idosos, promovidos por órgão públicos como prefeituras e universidades. No entanto, a participação e adesão a programas de atividades físicas pelos idosos dependem das características individuais, ambientais e das particularidades da atividade proposta, bem como do conhecimento dos benefícios que a atividade física proporciona. ${ }^{16}$

Portanto, detectar o tempo de prática no grupo de convivência e a quantidade de atividade física realizada pelos idosos é de fundamental importância, a fim de avaliar a contribuições dessas intervenções e a partir disso, sugerir propostas eficientes e coerentes em relação a tipo, duração, intensidade e frequência das atividades físicas propostas, com o objetivo de amenizar os efeitos decorrentes do envelhecimento. Poucos estudos têm sido realizados investigando a permanência dos idosos nos grupos de convivência, bem como a caracterização dos mesmos quanto ao nível de atividade física.

Sendo assim, o objetivo deste estudo foi verificar o nível de atividade física habitual e o tempo de prática em mulheres idosas praticantes 
de atividades físicas em um grupo de convivência de Florianópolis/SC.

\section{METODOLOGIA DO ESTUDO}

Este estudo é descritivo de corte transversa ${ }^{17} \mathrm{e}$ constituiu-se por mulheres idosas participantes de atividades físicas em um grupo de convivência localizado na cidade de Florianópolis, capital do Estado de Santa Catarina, Brasil. Florianópolis tem uma população idosa de 28.816, correspondendo a $8,4 \%$ de sua população ${ }^{18}$. Nele foram admitidas pessoas com idade igual ou superior a 60 anos de idade, do sexo feminino, com tempo de participação mínima no grupo de um ano nas atividades ginástica, recreação, dança, vôlei e hidroginástica, com frequência mínima de duas vezes semanais e duração de 40 a 50 minutos por sessão.

A amostra não probabilística constituiu-se de 234 idosas do sexo feminino, com idade igual ou superior a 60 anos.

Os dados foram coletados utilizando-se o questionário. No primeiro bloco do questionário, foram respondidas questões referentes às características sócio-demográficas - idade e escolaridade -, assim como questões referentes ao estado de saúde: satisfação com a saúde, doenças e uso de medicamentos. O segundo bloco foi composto por questões do Questionário Internacional de Atividades Físicas (IPAQ) forma longa, adaptado para idosos e considerado o mais indicado para discriminar níveis gerais de atividades físicas em idosos, com boa estabilidade de medidas e precisão ${ }^{15}$. Ele mensura o tempo realizado em atividades físicas moderadas e/ou vigorosas nos domínios do trabalho, transporte, atividades domésticas e de lazer durante uma semana usual. Para categorizar o nível de atividade física, foram consideradas menos ativas as idosas que, no somatório das atividades físicas nos diferentes domínios, despendiam menos de 150 minutos em atividades físicas moderadas ou vigorosas por semana, e mais ativas as idosas que obtiveram um escore igual ou superior a 150 minutos semanais. ${ }^{19}$ Para a classificação dos problemas de saúde referidos pelas idosas, utilizou-se a Classificação Estatística Internacional de Doenças e Problemas Relacionados à Saúde em sua décima revisão, volume I (CID - 10).

Para tanto, neste estudo foi utilizado o termo atividade física como qualquer movimento corporal, produzido pelos músculos-esqueléticos, que resulta em gasto energético maior do que os níveis de repouso. ${ }^{20}$

O tempo de prática (TP) é uma variável do estudo relacionada ao tempo (anos completos) em que as idosas praticam atividades físicas regularmente no grupo de convivência selecionado para este estudo. Para tanto, foram consideradas como iniciantes as idosas que estavam começando as atividades no grupo ou as que ainda não tinham completado um ano de participação nas atividades físicas do grupo, independentemente de terem feito atividades físicas em outros lugares anteriormente. A classificação foi feita considerando os anos completos de participação no grupo de convivência. Os dados referentes a esta variável foram obtidos por meio de entrevista individual.

Dentre os procedimentos para a coleta de dados, realizou-se um treinamento para a capacitação na aplicação do questionário internacional de atividades físicas (IPAQ), que serviu para orientar os procedimentos dos entrevistadores de forma a homogeneizar as condutas e assegurar uma boa compreensão e entendimento do instrumento, minimizando, assim, as interpretações equivocadas e diferenciadas que pudessem comprometeros resultados.

As idosas assinaram o termo de consentimento livre e esclarecido e os procedimentos metodológicos aplicados foram aprovados pelo Comitê de Ética em Pesquisa com Seres Humanos da Universidade Federal de Santa Catarina, sob o número 050-05.

Os dados considerando as diferentes variáveis do estudo foram analisados por meio do programa estatístico SPSS 12.0, utilizando-se dos procedimentos da estatística descritiva: média e desvio padrão. Foram calculadas também frequências relativas e frequências absolutas. 


\section{RESULTADOS}

As mulheres idosas participantes do estudo tinham uma média de idade de 69,52 $\pm 5,51$ anos, com idades variando entre 60 e 84 anos. Houve predominância de idosas com idades entre 7074,9 anos (29,5\%), seguidas de idosas de 65-69,9 anos $(28,6 \%)$. Em relação à escolaridade, predominaram idosas com ensino fundamental $(34,6 \%)$. O estado subjetivo de saúde é bastante variável entre as pessoas idosas e depende de suas experiências pessoais, nível de instrução e conhecimento, objetivos e mecanismos utilizados para enfrentar as decepções e os fracassos ao longo de suas vidas. Neste estudo, observou-se que a maioria (76,5\%), estava satisfeita/muito satisfeita com a saúde atual. Entre as idosas, as doenças foram autorreferidas e $90,6 \%$ apresentam algum tipo de doença, sendo que as ligadas ao aparelho circulatório foram as de maior porcentagem. $\mathrm{O}$ uso de medicamentos foi relatado por $86,8 \%$ das idosas e, destas, a maioria $(59,8 \%)$ faz uso diário de um a três medicamentos diferentes. Ao categorizar-se o nível de atividade física em dois níveis, observou-se uma predominância de idosas mais ativas $(82,1 \%)$ sobre as menos ativas $(17,9 \%)$ com valores médios em min/sem de 368,16 e 114,40 , respectivamente. Possivelmente, os percentuais de idosas mais ativas foram maiores no presente estudo em função de suas características particulares, ou seja, de ser um grupo específico de convivência que participa de atividades físicas regulares.

O IPAQ tem sido utilizado como um dos instrumentos de mensuração do nível de atividade física em grandes grupos populacionais, boa precisão e de baixo custo. Os valores em frequência relativa e absoluta, média, desvio padrão e mediana dos diferentes domínios considerando todas as participantes do estudo (n total) e apenas as idosas que realizaram atividade física de intensidade moderada e/ou vigorosa com duração mínima de dez minutos contínuos para cada domínio (n parcial) estão apresentados na Tabela 1.

Tabela 1 - Resultado dos diferentes domínios que compõem o IPAQ representados por meio da frequência (n), percentual (\%), média, desvio padrão (DP) e mediana da amostra total e parcial das idosas. Florianópolis, SC. 2008.

\begin{tabular}{lcccc}
\hline \multicolumn{1}{c}{$\begin{array}{c}\text { n } \\
\text { Domínios do IPAQ }\end{array}$} & Total & Total & Média $( \pm \mathrm{DP}) \mathrm{min} / \mathrm{sem}$ & Mediana $(\mathrm{min} / \mathrm{sem})$ \\
\hline AF Trabalho & 234 & 100 & $6,132( \pm 37,038)$ & 0 \\
AF Transporte & 234 & 100 & $67,618( \pm 75,924)$ & 40 \\
AF Domésticas & 234 & 100 & $67,179( \pm 95,557)$ & 30 \\
AF Lazer, Recreação & 234 & 100 & $181,979( \pm 124,189)$ & 140 \\
Tempo Sentada & & & & \\
Transporte & 234 & 100 & $156,915( \pm 133,211) \mathrm{min} / \mathrm{sem}$ & $130 \mathrm{~min} / \mathrm{sem}$ \\
Dia de Semana & 234 & 100 & $382,432( \pm 128,948) \mathrm{min} / \mathrm{dia}$ & $382,5 \mathrm{~min} / \mathrm{dia}$ \\
Fim de Semana & 234 & 100 & $461,521( \pm 122,153) \mathrm{min} / \mathrm{dia}$ & $470 \mathrm{~min} / \mathrm{dia}$ \\
\hline & $\mathrm{n}$ & & & \\
$\quad$ Domínios do IPAQ & Parcial & $\%$ Parcial & Média $( \pm \mathrm{DP}) \mathrm{min} / \mathrm{sem}$ & Mediana $(\mathrm{min} / \mathrm{sem})$ \\
\hline AF Trabalho & 15 & 6,4 & $91,842( \pm 105,74)$ & 50 \\
AF Transporte & 169 & 72,2 & $91,598( \pm 74,908)$ & 70 \\
AF Domésticas & 139 & 59,4 & $111,489( \pm 101,1)$ & 70 \\
AF Lazer, Recreação & 234 & 100 & $181,979( \pm 124,189)$ & 140 \\
Tempo Sentada & & & & \\
Transporte & 227 & 97 & $161,753( \pm 132,320) \mathrm{min} / \mathrm{sem}$ & $136 \mathrm{~min} / \mathrm{sem}$ \\
Dia de Semana & 234 & 100 & $382,432( \pm 128,948) \mathrm{min} / \mathrm{dia}$ & $382,5 \mathrm{~min} / \mathrm{dia}$ \\
Fim de Semana & 234 & 100 & $461,521( \pm 122,153) \mathrm{min} / \mathrm{dia}$ & $470 \mathrm{~min} / \mathrm{dia}$ \\
\hline
\end{tabular}


Considerando o total de idosas participantes do estudo, a média de atividade física habitual do grupo foi de 322,62 min/sem. O domínio no qual o dispêndio de tempo numa semana normal/ usual foi maior, apresentando uma média semanal de 181,97 minutos, foi no envolvimento em atividades de lazer, recreação e exercícios físicos. Provavelmente, tal predominância ocorra em função da característica do grupo em estudo, ou seja, um grupo de convivência em que as idosas participam regularmente de atividades físicas.

Quando se analisaram os domínios separadamente, considerando somente as idosas que realizaram atividade física com duração mínima de dez minutos contínuos (n parcial), pode-se observar que a atividade física no trabalho, que inclui as atividades que as idosas executam no seu trabalho remunerado ou voluntário, é o domínio ao qual as idosas dedicam menos tempo durante uma semana normal/usual. Neste estudo, 45,72\% (107) realizam algum tipo de trabalho remunerado e/ou voluntário; destas, a maioria, representada por $85,9 \%(92$,$) realiza$ atividades leves como pintura, costura, bordados e artesanatos em geral, enquanto $14,01 \%$ (15) se envolvem em atividades moderadas.

No domínio da atividade física como meio de transporte, observou-se que 27,8\% não utilizam a atividade física como meio de transporte para se deslocar de um lugar para o outro, enquanto a maioria $(72,2 \%)$ utiliza este meio, caminhando $(97,6 \%)$ ou andando de bicicleta $(2,3 \%)$ como forma de deslocamento.

Nas atividades físicas realizadas no âmbito doméstico com duração mínima de dez minutos contínuos, constatou-se que 40,6\% não realizam tais atividades, enquanto $59,4 \%$ se dedicam às tarefas domésticas; destas, a maioria se envolve em atividades físicas com intensidade moderada e apenas $1,7 \%$ em intensidade vigorosa, totalizando uma média de $111,48 \mathrm{~min} / \mathrm{sem}$. e mediana de 70 $\mathrm{min} / \mathrm{sem}$.
Em relação ao domínio que considera atividades físicas de recreação, lazer, esporte e exercício físico, destaca-se que todas as idosas participam destas atividades semanalmente com duração mínima de dez minutos contínuos, totalizando um tempo médio semanal de 181,97 min, com valor da mediana igual a $140 \mathrm{~min} /$ sem. Destas, $47 \%$ adotaram a caminhada como exercício físico no tempo livre, atingindo uma média de $72,69 \mathrm{~min} / \mathrm{sem}$. As atividades vigorosas, mais especificamente a musculação, foram citadas por apenas $0,85 \%$ das idosas, enquanto as atividades moderadas, predominantemente a ginástica e a hidroginástica, foram realizadas por todas (100\%), com duração média de $110,38 \mathrm{~min} / \mathrm{sem}$.

Ao analisarmos a frequência semanal de realização das atividades físicas de recreação, lazer, esporte e exercício físico, observou-se que a caminhada com uma frequência $\geq 3$ dias na semana é realizada por $24,8 \%$ das idosas, enquanto $23,1 \%$ realizam a caminhada como atividade física com uma frequência $\leq 2$ dias na semana. As atividades físicas moderadas são praticadas por $63,7 \%$ das idosas com uma predominância sobre a realização de $\leq 2$ dias semanais, enquanto $36,3 \%$ realizam atividades físicas de intensidade moderada com uma frequência semanal $\geq 3$ dias semanais. As atividades físicas vigorosas são realizadas por uma minoria com uma frequência semanal $\leq 2$ dias na semana.

Ao observarmos os quatro domínios que compõem o IPAQ (figura 1), os resultados indicam que o período de tempo $(\mathrm{min} / \mathrm{sem}) \mathrm{de}$ atividades moderadas e vigorosas realizadas por pelo menos dez minutos contínuos, apresentaram uma predominância das atividades físicas de lazer e recreação (42,01\%), seguidas das atividades físicas como meio de transporte $(30,33 \%)$, atividades físicas no âmbito doméstico (24,95\%) e em menor percentual, a realização de atividades físicas no trabalho (2,68\%). 


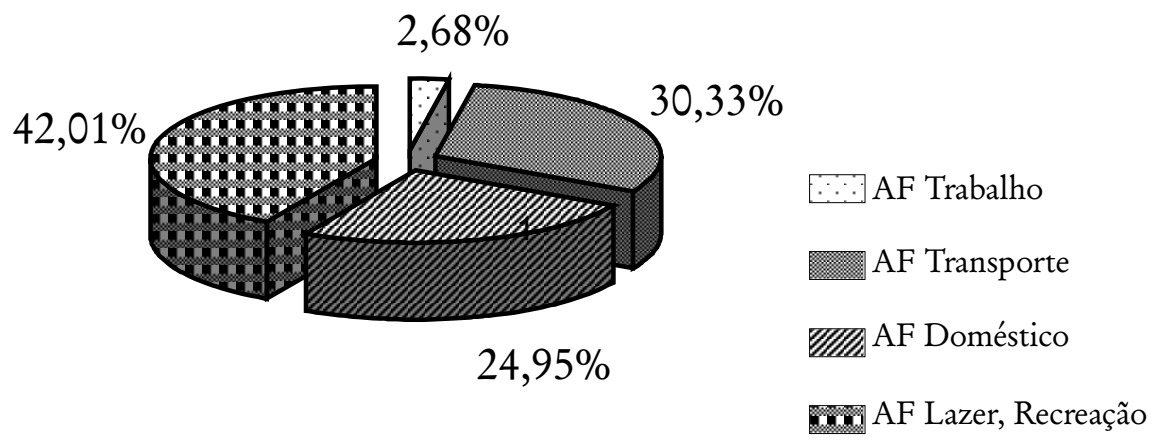

Figura 1 - Valores em percentuais dos diferentes domínios que compõem o IPAQ, considerando os idosos que fazem mais de 10 minutos de atividades contínuas. Florianópolis, SC. 2008.

O tempo sentado é analisado pelo IPAQ como um indicativo de sedentarismo. Este domínio "tempo sentado" está relacionado ao tempo que as idosas permanecem sentadas em diferentes locais, durante a semana e no fim de semana. Também foi incluído neste domínio o tempo gasto sentado durante o transporte em ônibus, carro e outros veículos de transporte. Todas as idosas deste estudo despendem tempo na posição sentada durante a semana e no fim de semana, porém durante o final de semana, a média é de 461,5 min/dia, enquanto que durante a semana a média é de $382,4 \mathrm{~min} /$ dia. Portanto, nos finais de semana, o tempo gasto sentado é maior do que em dias da semana, com uma diferença média de 79,0 min/dia. Quando se analisou o tempo sentado destas idosas durante o transporte em veículos a fim de se deslocar de um lugar para o outro, constatou-se que $97 \%$ delas gastam em média 161,7 $\mathrm{min} / \mathrm{sem}$ de tempo sentadas.

Nota-se, conforme tabela 2, que do tempo gasto sentado (horas) relatado pelas idosas, a maioria delas permanece de 5,01 a 10 horas sentada por dia, tanto nos dias da semana $(67,1 \%)$, quanto nos finais de semana $(78,2 \%)$. Esta tendência também foi observada quando analisamos juntamente o tempo sentado durante os dias da semana e os dias do final de semana (76,5\%). De acordo com os relatos ao longo das entrevistas, as idosas passam a maior parte deste tempo sentado realizando trabalhos manuais, assistindo televisão ou recebendo visitas. 
Tabela 2 - Valores percentuais do tempo que as idosas permanecem sentadas (horas) durante a semana, final de semana e ambos (total). Florianópolis, SC. 2008.

\begin{tabular}{lccc}
\hline Tempo (horas) & Dia da semana & Fim de semana & Total \\
\hline & $\%$ & $\%$ & $\%$ \\
$\leq 5$ & 28,2 & 10,7 & 19,7 \\
$5,01-10$ & 67,1 & 78,2 & 76,5 \\
$10,01-15$ & 4,7 & 11,1 & 3,8 \\
\hline
\end{tabular}

Para o tempo de prática, foram considerados os anos completos de participação no grupo de convivência em estudo, conforme tabela 3. De acordo com os dados da tabela 3 , as idosas que fizeram parte deste estudo apresentaram um tempo médio de participação no grupo de 7,35 $( \pm 5,34)$ anos. Observou-se uma predominância
$(35,9 \%)$ de idosas que praticam atividades físicas no grupo com uma média de 2,99 anos de prática. Pode-se constatar também que, quanto maior é a idade cronológica, maior é o tempo de prática em atividades físicas das idosas no grupo especificado, e que com o aumento da idade cronológica, as idosas tendem a diminuir o tempo semanal dedicado às atividades físicas.

Tabela 3 - Valores em frequência, percentual, média e desvio padrão (DP) da idade e do tempo de prática (TP) das idosas classificadas de acordo com o tempo de participação no grupo de convivência. Florianópolis, SC. 2008.

\begin{tabular}{ccccc}
\hline Classificação & $\mathrm{n}$ & $\%$ & Idade média $( \pm \mathrm{DP})$ anos & TP média $( \pm \mathrm{DP})$ anos \\
\hline Iniciante & 19 & 8,1 & $66,53( \pm 4,58)$ & 0 \\
$1-5$ & 84 & 35,9 & $68,67( \pm 5,33)$ & $2,99( \pm 1,44)$ \\
$6-10$ & 66 & 28,2 & $70,03( \pm 5,46)$ & $8,06( \pm 1,35)$ \\
$11-15$ & 41 & 17,5 & $70,63( \pm 5,73)$ & $12,78( \pm 1,40)$ \\
$16-20$ & 24 & 10,3 & $71,50( \pm 5,52)$ & $17,29( \pm 1,33)$ \\
\hline
\end{tabular}

\section{DISCUSSÕES}

Em relação aos níveis de atividade física habitual, em estudos que avaliaram idosas residentes em Florianópolis/SC, utilizando o mesmo instrumento para mensurar o nível de atividade física (IPAQ), e adotaram os mesmos critérios para categorizá-la, foram encontrados os seguintes resultados: considerando 198 mulheres idosas com a média de idade de 73,6 anos $(\mathrm{DP}=5.9)$, participantes em 33 grupos de convivência para idosos $33,8 \%$ foram consideradas menos ativas e $66,2 \%$ mais ativas; ${ }^{21}$ e num total de 875 idosos avaliados acima de 60 anos de idade, $45 \%$ das idosas do sexo feminino foram consideradas menos ativas, enquanto $55 \%$, mais ativas. ${ }^{22}$ Portanto, as prevalências são maiores para idosos mais ativos, sendo que neste estudo o valor de prevalência foi maior $(82,1 \%)$ quando comparados com os estudos anteriormente citados. Diante disso, pode-se inferir que os idosos que participam de grupos de convivência possuem, em sua maioria, bons níveis de atividade física.

Ao observarmos os diferentes domínios, constatouse que há predominância das atividades físicas de lazer e recreação $(42,01 \%)$ sobre os demais domínios. Em idosos americanos, quando nos reportamos especificamente aolazer, no estudo realizado por Yusut et al., ${ }^{23}$ foi verificado que $37 \%$ dos homens e $24 \%$ das mulheres eram mais ativos no lazer. 
Já em estudos realizados com idosas participantes de grupos de convivência, os maiores percentuais foram encontrados para atividades físicas no âmbito doméstico. De acordo com o estudo realizado por Mazo, ${ }^{21}$ a prevalência de atividades físicas no âmbito doméstico foi de $40 \%$, enquanto no estudo de Tribess, ${ }^{24}$ encontrou-se uma prevalência para este domínio de 54,6\%.

Ao referir-se ao tempo sentado como um indicador de sedentarismo, observou-se em estudo realizado por Mazo, ${ }^{21}$ que o tempo médio sentado no transporte para idosas que participavam de grupos de convivência em Florianópolis/SC foi de $176 \mathrm{~min} / \mathrm{sem}$, enquanto o tempo médio durante a semana foi $421 \mathrm{~min} /$ dia e, nos finais de semana, $472 \mathrm{~min} / \mathrm{dia}$. Tribess ${ }^{24}$ encontrou que o tempo médio gasto sentado em um dia de semana foi $405 \mathrm{~min} / \mathrm{dia}$, valor inferior ao tempo despendido durante o final de semana (480 min/ dia). Portanto, ambos os estudos apresentam resultados semelhantes aos encontrados no presente estudo, sendo que este apresentou valores inferiores nos aspectos analisados.

No estudo realizado por Benedetti, ${ }^{22}$ quando analisado o tempo sentado total, foram encontrados os maiores percentuais $(58,11 \%)$ para as idosas que permaneceram um tempo de 5,0110 horas por dia nesta posição. Portanto, o estudo de Benedetti ${ }^{22}$ apresentou um percentual inferior quando comparado com o presente estudo em relação à permanência das idosas na posição sentada durante os dias da semana e dos finais de semana. De qualquer forma, o tempo que as idosas permanecem sentadas, em ambos os estudos, é relativamente alto e, segundo Lee e Skerrett, ${ }^{25}$ isso pode resultar em consequências que irão repercutir para maximizar os riscos de desenvolvimento de doenças crônicodegenerativas, agravar as condições médicas, além de contribuir para a diminuição da funcionalidade com a idade.

Quando nos reportamos ao tempo de prática, observou-se que a adesão em atividades físicas no grupo de convivência estudado, por um período de tempo longo, é pequena, e isso pode ter ocorrido em função de diversos fatores relacionados ao envelhecimento, sobretudo pelo acometimento e pelas limitações físicas decorrentes das doenças. A aderência está relacionada à percepção da importância da atividade física, que por sua vez influencia a frequência, a duração das sessões e o tempo de adesão a atividades físicas ${ }^{16}$. Assim, a compreensão da importância da atividade física parece ser um fator de destaque que influencia os idosos na adesão a atividades físicas.

Considerando o resultado de que os idosos, com o aumento da idade cronológica, apresentam uma tendência de diminuir o tempo semanal dedicado às atividades físicas, sugere-se que a atenção em relação às estratégias de intervenção oferecidas pelos programas de atividades físicas seja voltada aos idosos com idades mais avançadas.

\section{CONCLUSÃO}

De acordo com os resultados do estudo, no que se refere à prática de atividade física habitual realizada as idosas, em sua maioria $(82,1 \%)$, foram consideradas mais ativas. A média semanal do grupo dedicado à prática de atividade física foi de 322,62 minutos/semana. Na contribuição dos diferentes domínios da atividade física no dispêndio total em minutos por semana de atividades físicas, destacam-se com maior prevalência as atividades de lazer/recreação $(42,01 \%)$.

Em relação ao tempo de prática das idosas no grupo, observou-se que o tempo médio de participação foi de 7,35 $( \pm 5,34)$ anos. Destaca-se também que, com o aumento da idade cronológica, as idosas tendem a diminuir o tempo semanal dedicado às atividades físicas.

Além da implantação de programas de atividades físicas eficientes e direcionados a este público, principalmente para os idosos com idades mais avançadas, os programas devem ter como meta melhorar as capacidades físicas dos indivíduos e conscientizá-los sobre a importância da prática como um fator de melhoria da qualidade de vida e de adesão regular aos programas. 
Sugere-se que outros estudos sejam realizados com este mesmo enfoque, a fim de verificar, no longo prazo, os motivos e as principais barreiras de adesão aos programas de atividades físicas, assim como um acompanhamento periódico dos níveis de atividades físicas desta população.

\section{REFERÊNCIAS}

1. Spirduso WW. Dimensões físicas do envelhecimento. Barueri, São Paulo: Manole; 2005.

2. Okuma SS. O idoso e a atividade física. Campinas, São Paulo: Papirus; 2002.

3. American College of Sport Medicine- ACSM. Position stand on exercise em physical activities for older adults. Medicine and Science in Sports Exercise 1998; 30 (6): 992 -1008.

4. Organização Mundial da Saúde - OMS. Active ageing. A policy framework. A contribution of the World Health Organization to the Second United Nations World Assembly on Ageing. Madrid (Spain); 2002.

5. Shephard RJ. Exercício e envelhecimento. Rev. Bras Ciên Moviment 1991; 5(4):49-56.

6. Paffenbarger RS. Some interrelations of physical activity, physiological fitness, health, and longevity. In: Bouchard C, Shepard R, Stephens $\mathrm{T}$ (orgs) physical activity, fitness and health. International preceedings and consensua statement Champaign: Humam Kinetcs Publishers 1994. p.119-133.

7. Hallal PC, Victora CG, Wells JC, Lima RC. Physical inactivity: prevalence and associated variables in Brazilian adults. Med Sci Sports Exerc 2003; 35: 1894-900.

8. Barros MV, Nahas MV. Comportamentos de risco, auto-avaliação do nível de saúde e percepção de estresse entre trabalhadores da indústria. Rev. Saúde Pública 2001; 35: 554-63.

9. Feskanich D, Willett W, Colditz G. Walking and leisure-time activity and risk of hip fracture in postmenopausal women. JAMA 2002; 288 (18):2300-2306.

10. Tinetti ME. Factors associated wich serious injury during falls by ambulatory nursing home residents. J. Am. Geriatr. Soc. 1987; 35:644-648.

11. Mitnitski AB, Graham JJ, Mogilner AE, Rockwood K. Frailty, fitness and late-life mortality in relation to chronological and biological age. BMC Geriatrics 2002; 2:1-8.

12. Nnodim JO, Alexander NB. Assessing falls in older adults: A comprehensive fall evaluation to reduce fall risk in older adults. Geriatrics 2005; 60(10):24-28.
13. Going S, Williams D, Lohman T. Aging and body composition: biological changes and methodological issues. Exercise Sport Science Reviews 1995; 23:411-449.

14. Rexrode KM, Buring JE, Manson JE. Abdominal and total adiposity and risk of coronary heart disease in men. International Journal Obesity and Related Metabolic Disorders 2001; 25(7):1047-1056.

15. Benedetti TB, Mazo GZ, Barros MVG. Aplicação do questionário internacional de atividades físicas para avaliação do nível de atividades físicas de mulheres idosas: validade concorrente e reprodutibilidade teste-reteste. Rev . Bras. Ciên Moviment 2004; 12(1):25-34.

16. Alonso DO, Santos NB, Figueira Jr AJ. The Meaning of Physical Activity for the Elderly: Implications on Adherence. Medicine \& Science in Sports \& Exercise 2006; 38(5):42-50.

17. Thomas JR, Nelson JK. Métodos de pesquisa em atividade física. Porto Alegre: Artemed; 2002.

18. Instituto Brasileiro de Geografia e EstatísticaIBGE. Perfil dos idosos responsáveis pelos domicílios no Brasil, 2002; Disponível em: http://www.ibge.gov.br/home/presidencia/ noticias/25072002pidoso.pdf. [Acesso: 14 de Out. 2008].

19. Pate RR, et al. Physical activity and public health: a recommendation from the Centers for Disease Control and Prevention and the American College of Sports Medicine. Journal American Medical Association 1995; 273 (5):402-407.

20. Caspersen CJ, Powell KE, Christenson GM. Physical activity, exercise and physical fitness: definitions and distinctions for health-related research. Public Health Reports; 100(2):126-131.

21. Mazo GZ. Atividade física e qualidade de vida de mulheres idosas. [Tese de Doutorado]. Portugal: Universidade do Porto, Faculdade de Ciências de Desportos e de Educação Física; 2003.

22. Benedetti TB. Atividade física: uma perspectiva de promoção da saúde do idoso no Município de Florianópolis. [Tese de Doutorado]. 
Florianópolis (SC): Universidade Federal de Santa Catarina; 2004.

23. Yusut HR, Croft JB, Giles WA et al. Leisuretime physical activity among older adults. Archives of Internal Medicine, 1996; 156:1321-6.

24. Tribess S. Percepção da imagem corporal e fatores relacionados à saúde em idosas.
[Dissertação de Mestrado]. Florianópolis (SC): Universidade Federal de Santa Catarina; 2006.

25. Lee IM, Skerrett PJ. Physical activity and allcause mortality: what is the dose-response relation? Medicine and Science in Sports and Exercise 2001; 33 (6):459-471.

Recebido: 30/6/2009

Revisado: 07/7/2010

Aprovado: 30/7/2010 